\title{
Distributed Search in Railway Scheduling Problems
}

\author{
Montserrat Abril, Miguel A. Salido, Federico Barber \\ Dpto. Sistemas Informáticos y Computación, Universidad Politécnica de Valencia \\ Camino de Vera s/n, 46022, Valencia, Spain \\ \{mabril, msalido, fbarber\}edsic.upv.es
}

\begin{abstract}
Many problems of theoretical and practical interest can be formulated as Constraint Satisfaction Problems (CSPs). Solving a general CSP is known to be NP-complete; however, distributed models may take advantage of dividing the problem into a set of simpler interconnected sub-problems which can be more easily solved. The purpose of this paper is threefold: first, we present a technique to distribute the constraint network by means of selection of tree structures. Thus, the CSP is represented as a meta-tree CSP structure that is used as a hierarchy of communication by our distributed algorithm. Then, a distributed and asynchronous search algorithm (DTS) is presented. DTS is committed to solving the meta-tree CSP structure in a Depth-First Search Tree. Finally, an intra-agent search algorithm is presented. This algorithm takes into account the Nogood_message to prune the search space. We have focused our research on the railway scheduling problem which can be distributed by tree structures. We show that our distributed algorithm outperforms well-known centralized algorithms.
\end{abstract}

keywords: Distributed Constraint Satisfaction Problems, Tree Partition, Train Scheduling.

\section{Introduction}

Many real problems in Artificial Intelligence (AI) as well as in other areas of computer science and engineering can be efficiently modelled as Constraint Satisfaction Problems (CSPs), which can be solved using constraint programming techniques. Some examples of such problems include: spatial and temporal planning, qualitative and symbolic reasoning, diagnosis, decision support, scheduling, hardware design and verification, real-time systems and robot planning. Most of the work is focused on general methods for solving CSPs.

However, many of the problems solved by using centralized algorithms are inherently distributed. Some works are currently based on distributed CSPs (see special issue of Artificial Intelligence, Volume 161, 2005). Furthermore, many researchers are working on graph partitioning (Schloegel, Karypis, \& Kumar 2003). The main objective of graph partitioning is to divide the graph into a set of regions so that each region has roughly the same number of nodes and the sum of all edges connecting different regions is minimized.

Preprint submitted to Elsevier
Researchers are almost always interested in the size of the problems (nodes or edges), although a few studies have been made on the graph structure of the sub-problems induced by the partition (Miller 1986). For instance, one study seeks a node-separator whose induced graph is Hamiltonian.

Graph partitioning can also be applied to constraint satisfaction problems. Thus, we can use graph partitioning when dealing with large-scale CSPs to distribute the problem into a set of sub-CSPs. For instance, we can divide a CSP into several subCSPs so that constraints among variables of each sub-CSP are minimized (Salido \& Barber 2006).

Otherwise, a domain-dependent partition can be used. This requires a deeper analysis of the problem to be solved. In this paper, we also show that a domain-dependent partition obtains a more adequate distribution so that greater efficiency is obtained.

In this paper, we present two techniques for structuring and solving CSPs. To this end, the CSP is previously organized in a meta-tree CSP structure so that the original constraint graph is partitioned into trees that represent subproblems. Thus, the search algorithm carries out the search in each node in linear time (Freuder 1982), (Dechter \& Pearl 1987).

Our research also focuses on the railway scheduling problem. Railway traffic has increased considerably, which has created the need to optimize the use of railway infrastructures. This is, however, a hard and difficult task. Our aim is to model the railway scheduling problem as a Constraint Satisfaction Problem (CSP) and solve it using a distributed CSP solver. Due to the topological properties of the railway scheduling problem, the resultant CSP can be distributed in semi-independent sub-problems so that the solution can be solved easier.

In the following section, we summarize some definitions about CSPs. A tree partition method is presented in section 3. Our distributed algorithm for solving meta-tree CSP structures is presented in section 4 . We present our intraagent search method in section 5 . In section 6 , we describe the railway scheduling problem. An evaluation of our methods over real railway networks is presented in section 7. Finally, we summarizes our conclusions in section 8 . 


\section{Centralized, Distributed and Partitionable CSPs}

In this section, we present some basic definitions related to CSPs, which will be convenient for our purposes and will unify works from the constraint satisfaction community. Then, we present three ways of solving a CSP: as a centralized problem, as a partitionable problem and as a distributed problem.

A CSP consists of: a set of variables $X=$ $\left\{x_{1}, x_{2}, \ldots, x_{n}\right\}$; each variable $x_{i} \in X$ has a set $D_{i}$ of possible values (its domain); a finite collection of constraints $C=\left\{c_{1}, c_{2}, \ldots, c_{p}\right\}$ that restricts the values that the variables can simultaneously take.

A solution to a CSP is an assignment of values to all the variables so that all constraints are satisfied; a problem with a solution is termed satisfiable or consistent.

A binary constraint network is a network in which every constraint subset involves at most two variables. In this case, the network can be associated with a constraint graph, where each node represents a variable and the arcs connect nodes whose variables are explicitly constrained (Dechter 1992).

A meta-tree CSP structure is a tree whose nodes are composed by trees. Thus, we will refer to the main tree as metatree CSP structure and to each individual tree as singletree. We will define each node of the meta-tree CSP structure as meta-node and each individual and atomic node of the trees as single-node. It can be deduced that each metanode corresponds to a single-tree. Each constraint between two single-nodes of different meta-nodes is called interconstraint. Each constraint between two single-nodes of the same meta-node is called intra-constraint.

Partition : A partition of a set $C$ is a set of disjoint subsets of $C$ whose union is $C$. The subsets are called the blocks of the partition.

Distributed CSP: A distributed CSP (DCSP) is a CSP in which the variables and constraints are distributed among automated agents (Yokoo \& Hirayama 2000).

Each agent has some variables and attempts to determine their values. However, there are inter-constraints and the value assignment must also satisfy them. In our model, there is a set of agents and each agent knows the set of constraints and the domains of variables involved in these constraints.

\section{Partition of CSPs}

There are many ways to solve a CSP. However, these problems can be classified into three categories: centralized problems, distributed problems, and partitionable problems.

- A CSP is a centralized CSP when there are no privacy/security rules between parts of the problem, and all knowledge about the problem can be gathered into one process. It is commonly recognized that centralized CSPs must be solved by centralized CSP solvers. Many problems are represented as typical examples to be modelled as a centralized CSP and solved using constraint programming techniques. Some examples are: sudoku, n-queens, map coloring, etc.

- A CSP is a distributed CSP when the variables, domains and constraints of the underlying network are distributed among agents. This distribution is carried out due to many factors: constraints may be strategic information that should not be revealed to competitors, or even to a central authority; a failure of one agent can be less critical and other agents might be able to find a solution without the failed agent. Examples of such systems are sensor networks, meeting scheduling, web-based applications, etc.

- A CSP is a partitionable CSP when the global problem can be divided into smaller problems (sub-problems) which must be coordinated to find the solution to the global problem. For example, the search space of a CSP can be divided into several regions, and a solution is found by using parallel computing.

Given these three categories, we can conclude that a distributed CSP cannot be solved by centralized techniques. However, can a centralized CSP be solved by distributed techniques? The answer is 'yes' if the CSP is partitionable and the size of the problem is high enough to decompose into a set of subproblems.

Real problems usually imply models with a great number of variables and constraints, causing dense networks of inter-relations. Problems of this kind can be handled as a whole only at overwhelming computational cost. Thus, it could be an advantage to decompose problems of this kind into several simpler interconnected sub-problems which can be more easily solved.

For instance, the map coloring problem is a typically centralized problem. The goal of a map coloring problem is to color a map so that regions sharing a common border have different colors. Let's suppose that each country of Europe must be colored. Figure 1 (1) shows a colored portion of Europe. This problem can be solved by a centralized CSP solver. However, if the problem is to color each region of each country of Europe (Spain, Figure 1(3); France, Figure $1(4)$ ), it is easy to see that the problem can be partitioned into a set of sub-problems that are grouped by clusters. This problem can be solved as a distributed problem, even when the problem is not inherently distributed.

A map coloring problem can be solved by first converting the map into a graph where each region is a vertex and an edge connects two vertices if and only if the corresponding regions share a border. In our problem of coloring the regions of each country of Europe, it can be observed that the corresponding graph maintains clusters representing each country (Spain, Figure 1(3); France, Figure 1(4)). Thus, the problem can be solved in a distributed way.

\section{Why Tree Partition?}

As Rina Dechter states in (Dechter 1992), a problem is considered easy when it admits a solution in polynomial time. In the context of constraint networks, a problem is easy if an algorithm like backtracking can solve it in a backtrackfree manner, i.e., without dead-ends, thus producing a solution in linear time with regard to the number of variables and constraints. Theoretical research has identified topological features that determine this level of consistency and has yielded tractable algorithms for transforming some networks into backtrack-free representations. 


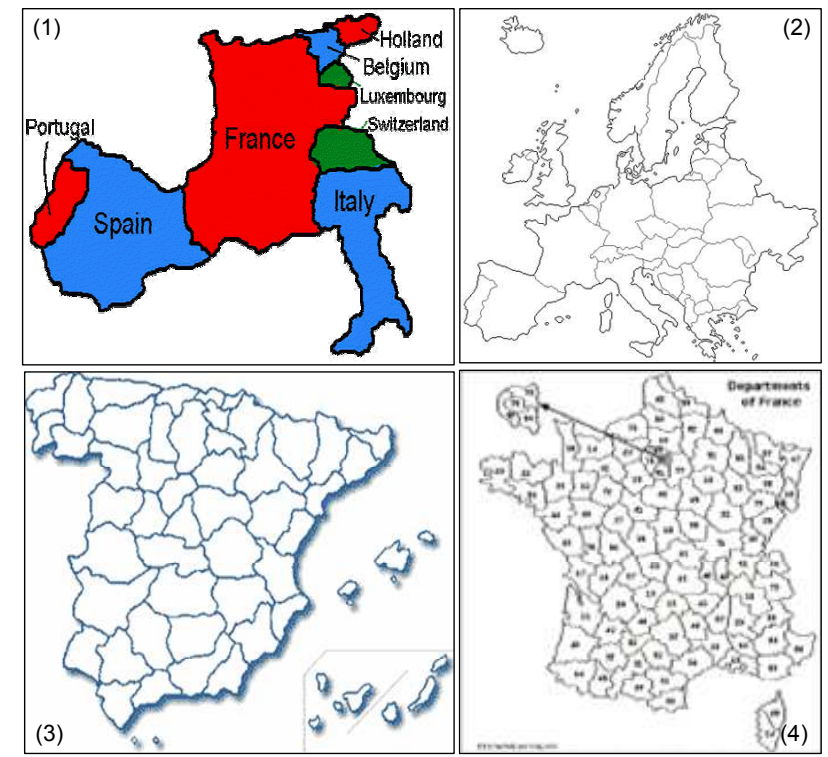

Figure 1: map coloring of Europe.

A main topological feature is centered on a graphical parameter called width, and the definitions are relative to the primal constraint graph. An ordered (primal) constraint graph is defined as one in which the nodes are linearly ordered to reflect the sequence of variable assignments executed by the backtracking algorithm. The width of a node is the number of arcs that connect that node to previous ones, the width of an ordering is the maximum width of all nodes, and the width of a graph is the minimum width of all orderings of that graph. It is known that only trees are widthone graphs (Freuder 1982). An ordered constraint graph is backtrack-free if the level of directional strong consistency along this order is greater than the width of the ordered graph. Thus, if the graph has width-one (i.e., it is a tree), a directional two-consistency is sufficient (Dechter 1992) to solve the problem in linear time.

\section{How to Convert a binary CSP into a Meta-Tree CSP Structure}

Any binary CSP can be translated into a meta-tree CSP structure. However, there are many ways to divide a graph into trees. Depending on user requirements, it may be desirable to obtain balanced single-trees, that is, each single-tree maintains roughly the same number of single-nodes; or it may be desirable to obtain single-trees in such a way that the number of edges connecting two single-trees are minimized.

Due to the complexity of finding the best partition, our technique finds an unbalanced tree partition in polynomial time ( $n^{2}$ in the worst case, where $n$ is the number of variables). It divides the problem into an undefined number of trees.

The TreePartition algorithm (Algorithm 1) divides the network graph $G$ into $k$ sub-graphs which are trees. The nodes and edges of graph $\mathrm{G}$ are the variables and constraints of the CSP, respectively. TreePartition randomly selects a root node that does not belong to another sub-graph, then the SearchTree function constructs a tree. This function recursively carries out a Depth First Search in graph $G$. The SearchTree function selects a new node $i$ which is connected with VarNode and whose inclusion in the present Tree does not introduce a cycle, that is, node $i$ is not connected with another node of the present Tree. Node $i$ is marked as visited; it indicates that this node already belongs to one tree. Tree construction finishes when either there are no unvisited nodes or the remainder nodes introduce cycles in the present tree. The TreePartition algorithm finishes when all nodes of $G$ belong to any sub-graph.

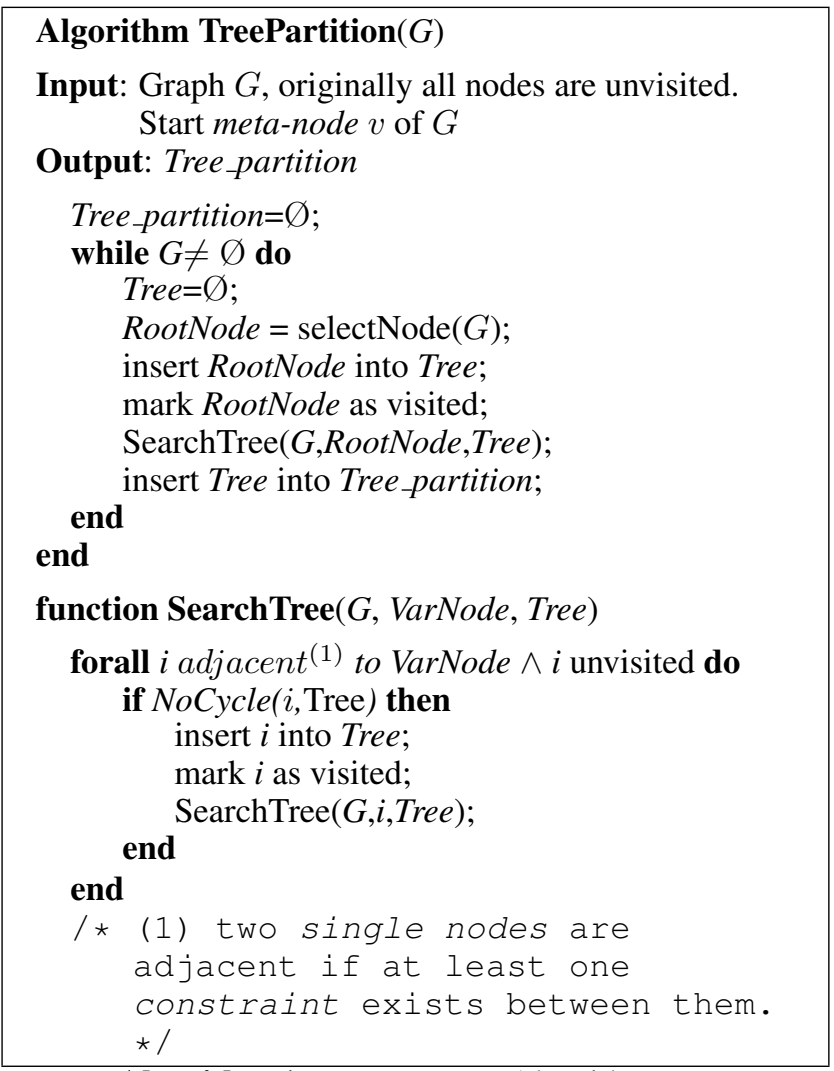

Algorithm 1: TreePartition Algorithm.

The next step is to build the meta-tree CSP structure with $k$ meta-nodes that will be studied by agents. This meta-tree CSP structure is used as a hierarchy to communicate messages between meta-nodes. The meta-tree CSP structure is built using Algorithm 2. The nodes and edges of graph $G$ are, respectively, the meta-nodes and inter-constraints obtained after the CSP partition. The root meta-node is obtained by selecting the most constrained meta-node. The MetaTreeCSPStructure algorithm then simply puts metanode $v$ into the meta-tree CSP structure (process $(v))$; it initializes a set of markers to indicate which vertices have been visited; it chooses a new meta-node $i$ and then calls MetaTreeCSPStructure $(G, i)$ recursively. If a meta-node has several adjacent meta-nodes, it would be equally correct to choose them in any order, but it is very important to delay the test for whether a meta-node is visited until the recursive 
calls for previous meta-nodes are finished.

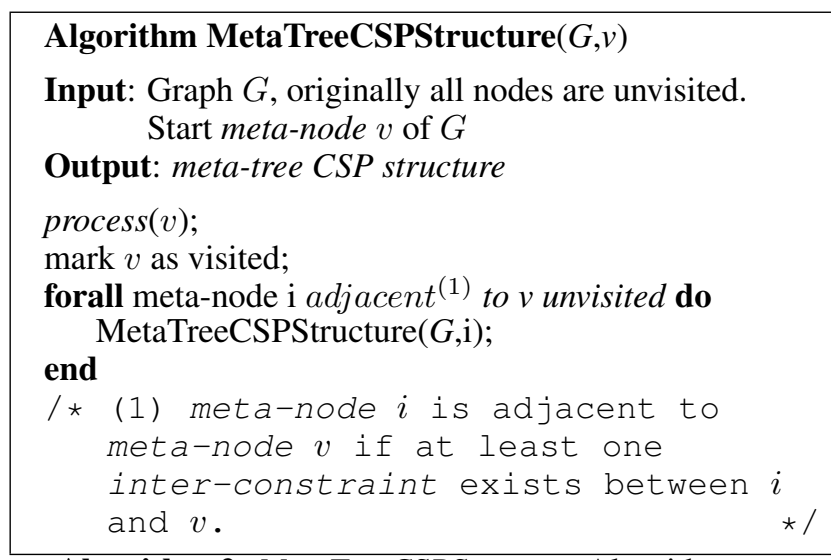

Algorithm 2: MetaTreeCSPStructure Algorithm.

Our aim is to solve CSPs by dividing the constraint graph by means of trees. Figure 3-1 shows a simple example of CSP. In Figure 3-2, this CSP has been divided into several trees and has been translated into a meta-tree CSP structure.

\section{DFSTreeSearch Algorithm (DTS)}

In the specialized literature, there are many works about distributed CSPs. In (Yokoo \& Hirayama 2000), Yokoo et al. present a formalization and algorithms for solving distributed CSPs. These algorithms can be classified as centralized methods, synchronous backtracking or asynchronous backtracking (Yokoo \& Hirayama 2000).

Our algorithm, called DFSTreeSearch (DTS), can be considered as a distributed and asynchronous algorithm. DTS is committed to solving the meta-tree CSP structure in a Depth-First Search Tree (DFS Tree) where the root metanode is composed of the most constrained single-tree (in the sense that this single-tree maintains a higher number of single-nodes). DFS trees have already been investigated as a means to boost search (Decher 2003). Due to the relative independence of nodes lying in different branches of the DFS tree, it is possible to perform search in parallel on these independent branches.

Once the variables are divided and arranged, the problem can be considered as a distributed CSP, where a group of agents manages each single-tree with its variables (singlenodes) and its constraints (intra-constraints). Each agent is in charge of solving its own sub-problem by means of any search method. Each sub-problem is composed of its CSP, which is subject to the variable assignment generated by the ancestor agents in the meta-tree CSP structure.

Thus, the root agent works on its sub-problem (root metanode). If the root agent finds a solution, then it sends the consistent partial state to its children agents in the meta-tree CSP structure, and all children work concurrently to solve their specific sub-problems knowing the consistent partial states assigned by the root agent. When a child agent finds a consistent partial state, it again sends this partial state to its children and so on. Finally, leaf agents try to find a solution to their own sub-problems. If each leaf agent finds a consistent partial state, it sends an OK message to its parent
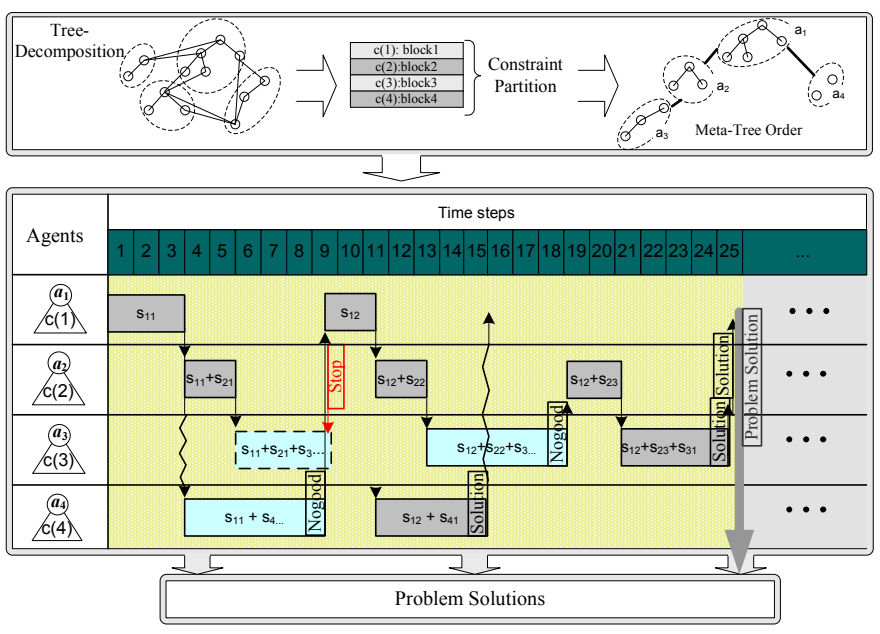

Figure 2: Execution of DFSTreeSearch Algorithm.

agent. When all leaf agents answer their parents with $\mathrm{OK}$ messages, a solution to the entire problem is found. When a child agent does not find a solution, it sends a Nogood message to the parent agent. The Nogood message contains the variables that empty the variable domains of the child agent. When the parent agent receives a Nogood message, it stops the search carried out by the children and tries to find a new solution taking into account the Nogood information and so on. If a parent agent finds a new solution, it will start the same process again sending this new solution to its children. Each agent works in the same way with its children in the meta-tree CSP structure. However, if the root agent does not find a solution, then DTS returns no solution found.

The top of Figure 2 shows our technique for partitioning a CSP into a meta-tree CSP structure. Then, DTS is carried out. Once the CSP is partitioned, the root agent $\left(a_{1}\right)$ starts the search process finding a partial solution. It sends this partial solution to its children. Agents that are brothers are committed to concurrently finding the partial solutions of their sub-problems. Each agent sends the partial problem solutions to its children agents. A problem solution is found when all leaf agents find their partial solution. For example, (state $\left.s_{12}+s_{41}\right)+\left(\right.$ state $s_{12}+s_{23}+s_{31}$ ) is a problem solution. The concurrence can be seen in Figure 2 in Time step 4 in which agents $a_{2}$ and $a_{4}$ are concurrently working. agent $a_{4}$ sends a Nogood message to its parent (agent $a_{1}$ ) in step 9 because it does not find a partial solution. Then, agent $a_{1}$ stops the search process of all its children and it finds a new partial solution which is sent to its children. Now, agent $a_{4}$ finds its partial solution, and agent $a_{2}$ works with its child, agent $a_{3}$, to find their partial problem solution. When agent $a_{3}$ finds its own partial solution, the global problem will be found. This happens in Time step 25.

\section{Example}

Figure 3 shows an example to analyze the behavior of DTS.

First, the constraint network of Figure 3(1) is partitioned into three trees and the DFS tree is built (Figure 3(2)). Agent 
$a$ finds its first partial solution $\left(X_{1}=1, X_{2}=1\right)$ and sends it to its children: agents $b$ and $c$ (see Figure 3(3)). This is a good partial solution for agent $c$ (Figure 3(4)); however this partial solution empties the $X_{3}$ variable domain. Thus, agent $b$ sends a Nogood message to its father $\left(\operatorname{Nogood}\left(X_{1}=1\right)\right)$ (Figure 3(5)). Then, agent $a$ processes the Nogood message, prunes its search space, finds a new partial solution $\left(X_{1}=2, X_{2}=2\right)$ and sends it to its children (Figure 3(6)). At this point in the process, agent $c$ sends a Nogood message to its father $\left(\operatorname{Nogood}\left(X_{1}=2\right)\right)$ because $X_{5}$ variable domain is empty (Figure 3(7)). Agent $a$ stops the search of agent $b$ (Figure 3(8)) and then processes the Nogood message, prunes its search space, finds a new partial solution $\left(X_{1}=3, X_{2}=3\right)$ and sends it to its children (Figure 3(9)). Since this last partial solution is good for both children, they respond with an OK message and the search ends (Figure 3(10)), and returns the solution presented in Figure 3(11).

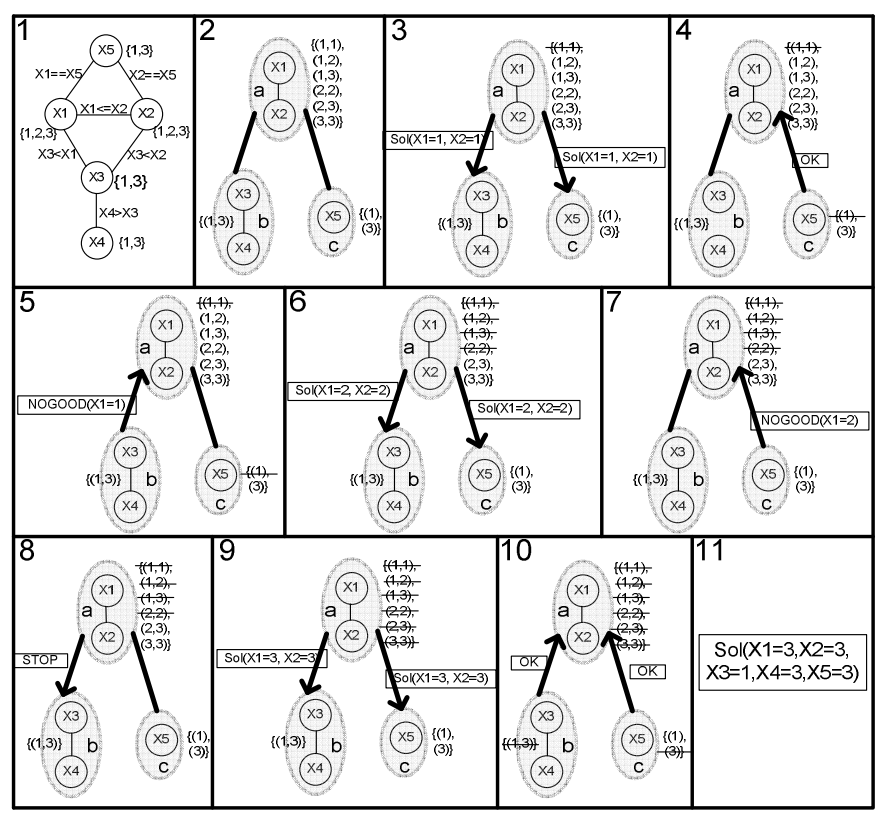

Figure 3: Example of DTS execution.

\section{Intra-agent search}

In section 4, we showed the distributed algorithm DTS for solving distributed CSPs, and we pointed out that each agent is in charge of solving its own sub-CSP by means of a search method. Agents are free to select their own search methods in order to find partial_solutions.

In this section, we propose a new algorithm to solve subCSPs with a tree structure: Tree Search Algorithm (TSA). This algorithm is based on the theorem presented in (Decher 2003): "Let $d$ be a width-1 ordering of a constraint tree $T$. If $T$ is arc-consistent relative to $d$, then the network is backtracking-free along $d$ ". Therefore, TSA finds a partial_solution in polynomial time. Furthermore, our algorithm is based on Nogood_message, which allows us to prune the search space.
TSA $(G$, Nogood_message $)$

Input: Constraint Graph $G=\{X, E\}$ where $\mathrm{E}$ is a set of constraints and $X=\left\{x_{1}, \ldots, x_{n}\right\}$ is a set of variables ordered according to $d=\left(x_{1}, \ldots, x_{n}\right)$; Nogood_message: set of inconsistent variables.

Output: partial_solution

if Nogood_message $==\varnothing$ then

$\mathrm{DAC}(\mathrm{G}) ; /$ * apply directional arc-consistency along $d . \quad \star /$

if $\forall X_{i}: D_{i} \neq \varnothing$ then else solution $\leftarrow$ assignValue $(1)$; end

return $N O \_S O L U T I O N$;

else

markVarNogood( $X$, Nogood_message); if $\left(\left(\exists x_{j}, x_{s} \in X: x_{j}, x_{s} \in\right.\right.$ Nogood_message and $s>j)$ and ( $\nexists x_{t} \in X:\left(x_{t} \in\right.$ Nogood_message $\wedge j<t<s)$ ) then set value $\left(x_{j}\right)$ restricted by $x_{s}$; reviewDAC $\left(x_{j}, x_{s}\right)$;

else

end reviewDAC $\left(x_{j}, x_{1}\right)$;

solution $\leftarrow$ NO_SOLUTION;

while solution $==$ NO_SOLUTION do $k \leftarrow j$; while $\left(k \geq 1\right.$ and $\left.D_{k}==\varnothing\right)$ do end $k \leftarrow$ previousVarNogood;

if $k<1$ then return $N O \_S O L U T I O N$; else

foreach $x_{i} \in X / j \leq i<n$ do end reset effects of $x_{i} \leftarrow \operatorname{value}\left(x_{i}\right)$;

solution $\leftarrow$ assignValue $(k)$;

if $\nexists x_{i} \in$ Nogood_message: $x_{i}$ changed $\operatorname{value}\left(x_{i}\right)$ then

solution $\leftarrow$ NO_SOLUTION; markVarNogood(X,Nogood_message); set value $\left(x_{j}\right)$ restricted by $x_{s}$; reviewDAC $\left(x_{j}, x_{s}\right)$; end end end

end

return solution;

Algorithm 3: Tree Search Algorithm.

\section{reviewDAC(startVar, endVar)}

if (parent (startVar) < endVar and review(parent( startVar), startVar,endVar)) then end reviewDAC(parent(startVar),endVar);

Algorithm 4: ReviewDAC Algorithm. 


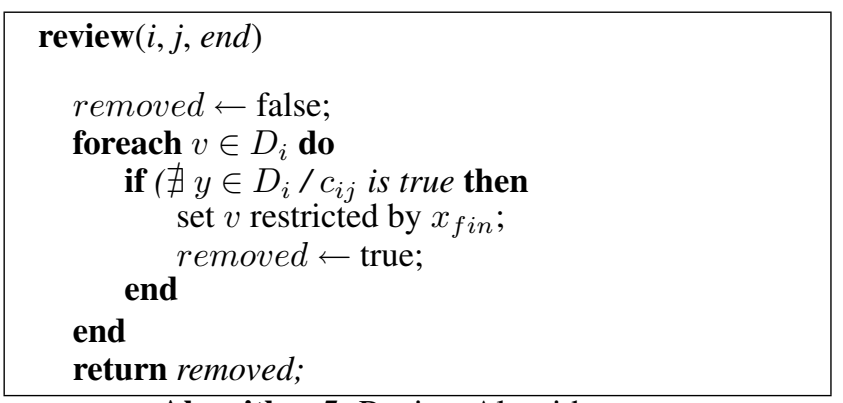

Algorithm 5: Review Algorithm.

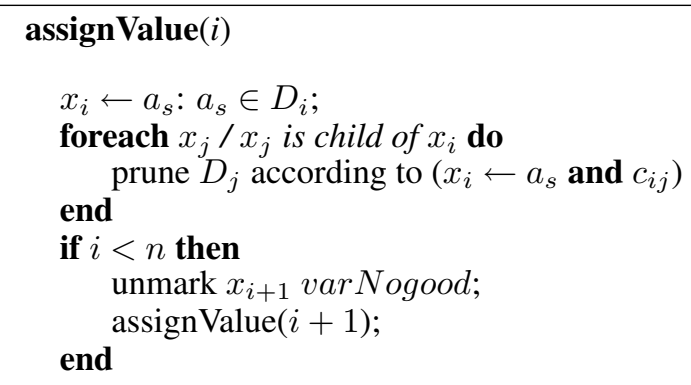

Algorithm 6: AssignValue Algorithm.

markVarNogood(Var, Nogood_message)

$$
\begin{aligned}
& x_{i} \leftarrow a_{s}: a_{s} \in D_{i} ; \\
& \text { foreach } x_{i} /\left(x_{i} \in \operatorname{Var} \text { and } x_{i} \in\right. \text { Nogood_message) } \\
& \text { do } \\
& \quad \text { mark } x_{i} \text { as } \operatorname{Var} N \text { ogood; } \\
& \quad x_{j} \leftarrow \text { parent }\left(x_{i}\right) \\
& \quad \text { while } x_{j} \neq \varnothing \text { do } \\
& \quad \text { mark } x_{j} \text { as } \operatorname{Var} \text { Nogood; } \\
& \quad x_{j} \leftarrow \operatorname{parent}\left(x_{j}\right) \\
& \quad \text { end } \\
& \text { end }
\end{aligned}
$$

\section{Algorithm 7: MarkVarNogood Algorithm.}

The algorithms 3, 4, 5, 6 and 7 show the pseudo-code of the Tree Search Algorithm (TSA). Algorithm 3 shows the main function of TSA and the others show the auxiliary functions. The main algorithm starts with a tree network structure and a width-1 ordering $d$. TSA has a different behavior for finding the first solution of the subproblem than for finding the rest of the solutions of the subproblem. To find the first solution, TSA carries out directional arc-consistency along $d$. If the network is directional arcconsistent, then TSA uses the algorithm assignValue(1) following the established order $d$ to ensure that no backtracking will be necessary. If the network is not directional arc-consistent, then the problem is not consistent and returns $N O-S O L U T I O N$.

If TSA receives a Nogood message and $X_{j}$ is the highest variable included in the Nogood message(according to ordering $d$ ), TSA bounds the search space by means of avoiding the domains of higher variables to $X_{j}$. Thus, any valid partial solution can be lost due to the fact that the removed search space is not consistent with the entire problem. We must take into account that no variable included in the Nogood has been assigned to any other value of its domain.

Once TSA receives a Nogood message, it labels all the variables involved in the Nogood message and the preceding variables, (according to $d$ ), as VarNogood. Then TSA carries out the algorithm reviewD $A C$ because a value corresponding to the variables involved in the Nogood message has been removed or bounded. To carry our this task, we must take into account two different cases:

- If the Nogood message maintains only one variable $\left(X_{j}\right)$ owned by the agent, then the assigned value of this variable must be removed and the algorithm reviewDAC must check the domains of the variables ranging between $X_{1}$ and $X_{j}$ to ensure that these variables guarantee directional arc-consistency. It is not necessary to check directional arc-consistency for the rest of the variables due to the fact that the value removed from variable $X_{j}$ does not affect to the rest of the variables.

- If the Nogood message maintains more than two variables, the current value of variable $X_{j}$ (the variable with the highest order, according to $d$, included in the Nogood message) will not be removed. However it will be labelled as bounded by the value of variable $X_{s}$ (the second variable with the highest order, according to $d$, involved in the Nogood message). In this case, the algorithm reviewDAC must check the domains of variables ranging between $X_{j}$ and $X_{s}$ since when to as $X_{s}$ changes its current value, the value of $X_{j}$ that is bounded by $X_{s}$ will be included in the domain again.

The algorithm reviewDAC only checks directional arcconsistency in the branch of the tree that is involved in the removal of a value of a variable, since this does not affect any other branch of the tree.

The following step in the search process is to backtrack to the highest variable involved in the $\operatorname{Nogood}$ message $X_{j}$. To this end, TSA searches the first variable with no empty domain from $X_{j}$, in decreasing order. If no variable is found, then TSA returns NO-SOLUTION. Otherwise, if a variable $X_{k}$ has no empty domain, then TSA unassigns the value of all the variables that are higher than $X_{k}$ according to $d$ ( $X_{k}$ included). Then, TSA calls algorithm assignValue to assign values to variables from $X_{k}$ to $X_{n}$. To prevent the new solution generating the same Nogood message, TSA searches for a solution with different value assignments in, at least, one of the variables involved in the previous Nogood message.

Next, we show an example of TSA execution. It is based on the DCSP shown in Figure 4. This figure shows a CSP divided into two sub-problems with tree structure.

According to the DTS algorithm and TSA, the process starts with the search for the first partial_solution in sub-problem 1. First, the TSA carries out a directional arc-consistency according to ordering $d=\left\{X_{1}, X_{2}, X_{3}, X_{4}, X_{5}, X_{6}\right\}$. This action prunes the domains of the variables $X_{1}$ and $X_{4}$ as shown in Figure 4. Then, sub-problem 1 has the partial_solutions shown in Figure 5. 

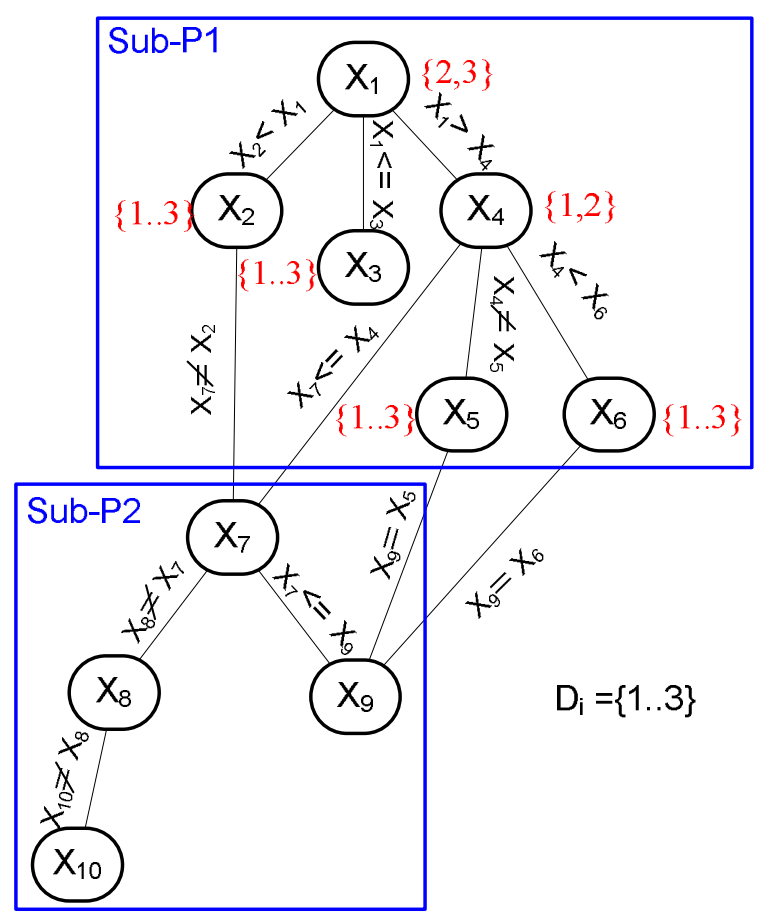

Figure 4: Example of DCSP with tree structure.

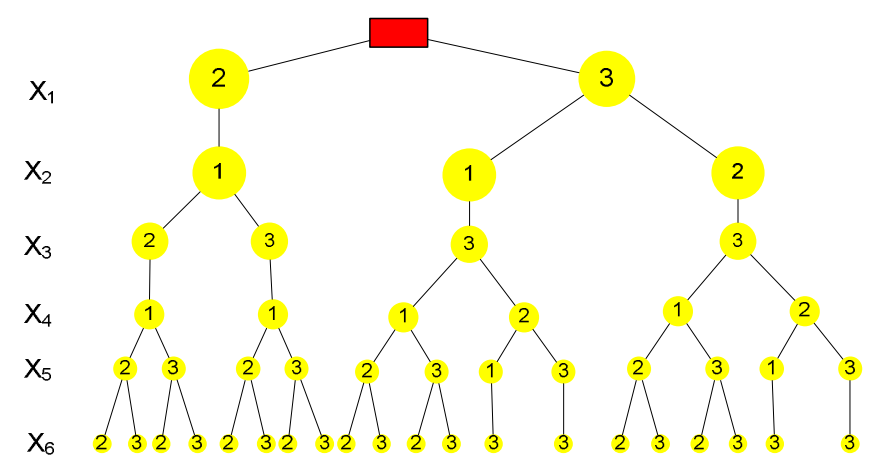

Figure 5: Set of valid partial_solutions of Sub-P1 (Figure 4) after directional arc-consistency.

Next, TSA assigns values to its variables. The first partial_solution included in the set of valid partial_solutions shown in Figure 5 is $\left(X_{1}=2\right.$, $\left.X_{2}=1, X_{3}=2, X_{4}=1, X_{5}=2, X_{6}=2\right)$. This first partial_solution is inconsistent with sub-problem 2 since assignments $X_{2}=1$ and $X_{4}=1$ empty the domain of $X_{7}$. Therefore, the agents that owns sub-problem 2 sends the Nogood message: ( $X_{2}=1, X_{4}=1$ to the other agent.).

When the agent that owns sub-problem 1 gets the Nogood message $\left(X_{2}=1, X_{4}=1\right)$, it marks the value of $X_{4}$ restricted by $X_{2}$. It causes the pruning of seven partial_solutions that are valid for sub-problem 1 but which are inconsistent with the global problem (see Figure 6). Next, this agent marks the variables $X_{4}, X_{2}$ and $X_{1}$ as varNogood and it searches for a varNogood variable that has an available value beginning with $X_{4}$. The found variable is $X_{1}$. Then this agent assigns new values beginning with $X_{1}$ and it finds a new partial_solution: $X_{1}=3, X_{2}=1, X_{3}=3, X_{4}=1, X_{5}=2, X_{6}=2$ (Figure 7). This partial_solution does not change any value of the variables in Nogood message. Therefore, TSA repeats the same process again: it marks the value of $X_{4}$ restricted by $X_{2}$. Now, this causes the pruning of three partial_solutions (see Figure 7). TSA again marks the variables $X_{4}, X_{2}$ and $X_{1}$ as varNogood. In this case, variable $X_{4}$ is the first varNogood variable with available values. Thus, TSA assigns new values beginning with $X_{4}$ and it obtains the partial_solution: $X_{1}=3$, $X_{2}=1, X_{3}=3, X_{4}=2, X_{5}=1, X_{6}=3$.

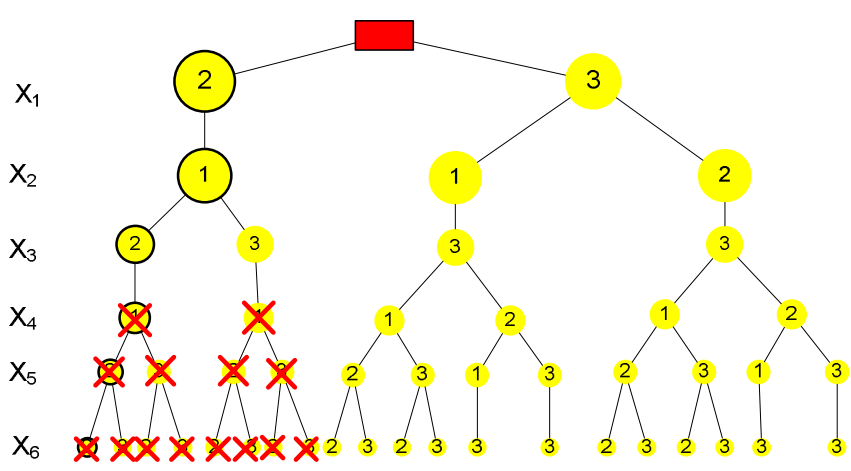

Figure 6: TSA pruning with Nogood-message $\left(X_{2}=1, X_{4}=1\right)$.

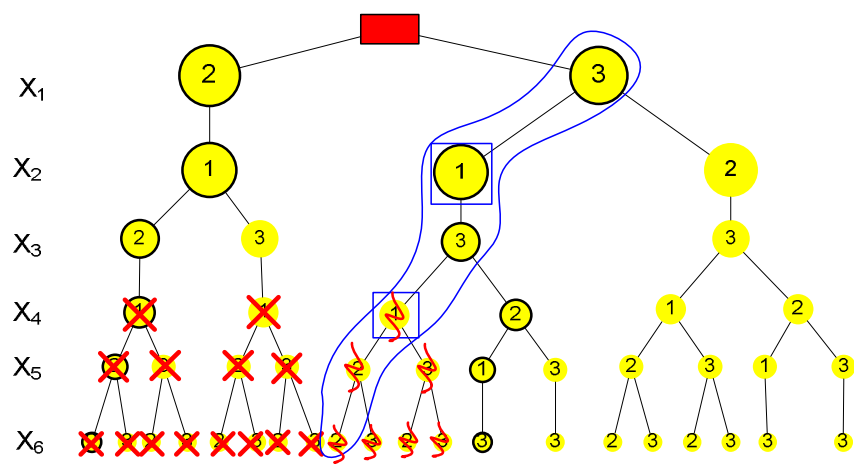

Figure 7: Second TSA pruning with Nogood-message $\left(X_{2}=1, X_{4}=1\right)$.

Again, the agent that owns sub-problem 2 sends a $\mathrm{No}$ good message $\left(X_{5}=1, X_{6}=3\right)$ since the last partial_solution of sub-problem 1 empties the domain of $X_{9}$. When the agent that owns sub-problem 1 gets this Nogood message, it marks the value of $X_{6}$ restricted by $X_{5}$, and it marks the variables $X_{5}, X_{6}$ and their ancestors as varNogood. Beginning with $X_{6}$, the first variable with available values is $X_{5}$. The value reassignment, beginning with $X_{5}$, obtains the partial_solution: $X_{1}=3, X_{2}=1, X_{3}=3, X_{4}=2, X_{5}=3, X_{6}=3$ (see Figure 8).

Finally, the last partial_solution of sub-problem 1 is consistent with sub-problem 2, for example with the assign- 
ment: $\left(X_{7}=2, X_{8}=1, X_{9}=3, X_{10}=2\right)$. Then, we have a global solution and the DTS algorithm finishes.

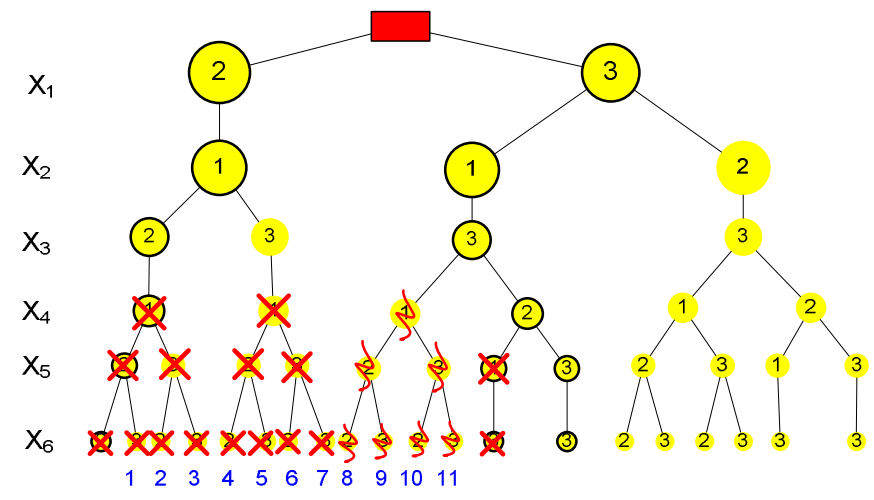

Figure 8: TSA pruning with Nogood-message $\left(X_{5}=1, X_{6}=3\right)$.

Figure 8 shows that thanks to the Nogood messages, TSA has pruned eleven partial_solutions of sub-problem 1 that are inconsistent with sub-problem 2.

\section{Railway Scheduling Problem}

Train timetabling is a difficult and time-consuming task, particularly in the case of real networks where the number of constraints and the complexity of constraints grow drastically. A feasible train timetable should specify the departure and arrival time of each train to each location of its journey, in such a way that the line capacity and other operational constraints are taken into account. Traditionally, train timetables are generated manually by drawing trains on a time-distance graph called a running-map. The train schedule is generated from a given starting time and is manually adjusted so that all constraints are met. High priority trains are usually placed first followed by lower priority trains. It can take many days to develop train timetables for a line, and the process usually stops once a feasible timetable has been found. The resulting plan of this procedure may be far from optimal.

A sample of a running map is shown in Figure 9, where several train crossings can be observed. A running map contains information regarding railway topology (stations, tracks, distances between stations, traffic control features, etc.) and the schedules of the trains that use this topology (arrival and departure times of trains at each station, frequency, stops, crossings, etc,). The names of the stations are presented on the left side of Figure 9, and the vertical line represents the number of tracks between stations (one-way or two-way). The horizontal line represents the time.

The literature of the 1960s, 1970s, and 1980s related to rail optimization was relatively limited. Compared to the airline and bus industries, optimization was generally overlooked in favor of simulation or heuristic-based methods. However, (Cordeau, Toth, \& Vigo 1998) point out greater competition, privatization, deregulation, and increasing computer speed as reasons for the more prevalent use of optimization techniques in the railway industry. Our review

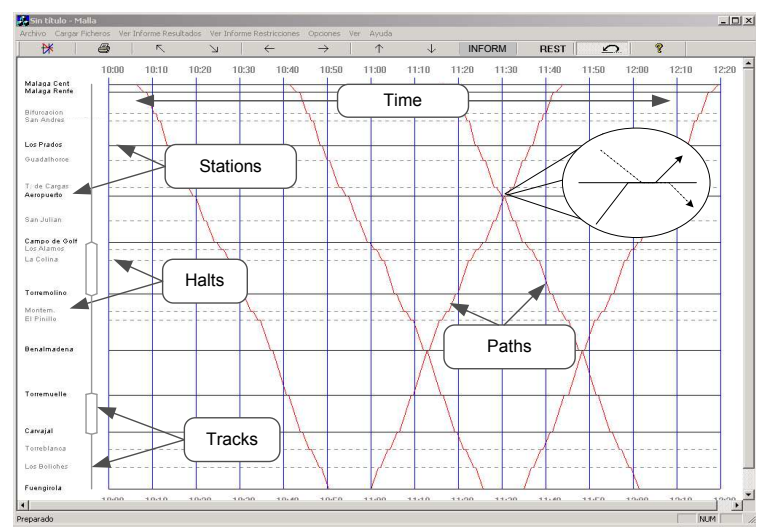

Figure 9: Example of a running-map

of the methods and models that have been published indicates that the majority of authors use models that are based on the Periodic Event Scheduling Problem (PESP) introduced by (Serafini \& Ukovich 1989). The PESP considers the problem of scheduling as a set of periodically recurring events under periodic time-window constraints. The model generates disjunctive constraints that may cause the exponential growth of the computational complexity of the problem depending on its size. (Schrijver \& Steenbeek 1994) have developed CADANS, a constraint programming- based algorithm to find a feasible timetable for a set of PESP constraints. The scenario considered by this tool is different from the scenario that we have used in this work; therefore, the results are not easily comparable. The train scheduling problem can also be modeled as a special case of the jobshop scheduling problem ((Silva de Oliveira 2001), (Walker \& Ryan 2005)), where train trips are considered jobs that are scheduled on tracks that are regarded as resources.

Our goal is to model the railway scheduling problem as a Constraint Satisfaction Problem (CSP) and to solve it using constraint programming techniques. However, due to the huge number of variables and constraints that this problem generates, a distributed model is developed to distribute the resultant CSP into semi-independent sub-problems so that the solution can be found efficiently.

\section{Variables and Constraints in the Railway Scheduling Problem}

The variables of the railway scheduling problem are the arrival and departure times of trains at stations. The domain of the variables is the time with a granularity of minutes. There are three groups of scheduling rules in our railway scheduling problem: traffic rules, user requirements rules and topological rules. A valid running map must satisfy the above rules. These scheduling rules can be modelled using the following constraints, where variable $T A_{i, k}$ represents that train $i$ arrives at station $k$ and the variable $T D_{i, k}$ means that train $i$ departs from station $k$ :

1. Traffic rules guarantee crossing and overtaking operations. We assume two trains ( $i$ and $j$ ) going in opposite 
directions between stations $k$ and $k+1$. The main constraints to take into account are:

- Reception time constraint. There exists a given time to detour a train back from the main track so that crossing or overtaking can be performed (RecT).

$$
\left(T A_{i, k}+\operatorname{Rec} T_{i}<T A_{j, k}\right) \vee\left(T A_{j, k}+\operatorname{Rec} T_{j}<T A_{i, k}\right)
$$

- Expedition time constraint. There exists a given time to put a train back on the main track so that crossing or overtaking can be performed (ExpT).

$$
\left(T D_{i, k}+\operatorname{Exp} T_{i}<T D_{j, k}\right) \vee\left(T D_{j, k}+\exp T_{j}<T D_{i, k}\right)
$$

- Crossing constraint: Any two trains going in opposite directions must not simultaneously use the same oneway track.

$\left(T D_{i, k}+T_{i,\left(k \_k+1\right)}<T D_{j, k+1}\right) \wedge\left(T D_{i, k}<T D_{j, k+1}+T_{j,\left(k+1 \_k\right)}\right)$ $\checkmark$

$\left(T D_{i, k}+T_{i,\left(k_{-} k+1\right)}>T D_{j, k+1}\right) \wedge\left(T D_{i, k}>\left(T D_{j, k+1}+T_{j,\left(k+1 \_k\right.}\right)\right.$

- Overtaking constraint: Two trains ( $i$ and $s$ ) going at different speeds in the same direction can only overtake each other at stations.

$\left(T D_{i, k}<T D_{s, k}\right) \wedge\left(T D_{i, k}+T_{i,(k-k+1)}<T D_{s, k}+T_{s,\left(k \_k+1\right)}\right)$

$\left(T D_{i, k}>T D_{j, k}\right) \wedge\left(T D_{i, k}+T_{i,\left(k \_k+1\right)}>\left(T D_{s, k}+T_{s,\left(k \_k+1\right)}\right)\right.$
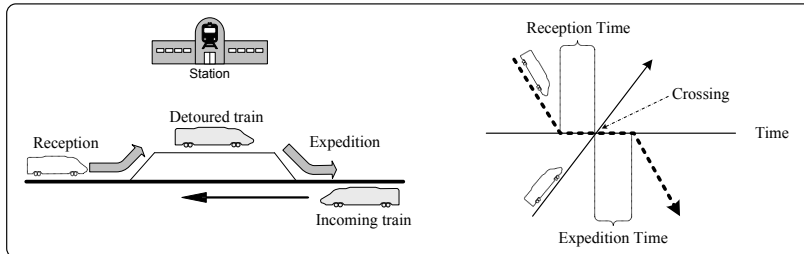

Figure 10: Constraints related to crossing and overtaking in stations

2. User Requirements: The main constraints due to user requirements are:

- Type and Number of trains going in each direction to be scheduled.

- Path of trains: Locations used and Stop time for commercial purposes in each direction.

$$
T D_{i, k}=T A_{i, k}+\text { StopTime }_{i, k}
$$

- Scheduling frequency. Train departure must satisfy frequency requirements in both directions. It could be a fixed time (1) or a time interval $($ Freq $\pm \delta)(2)$. Frequency is a very tight constraint and is only sometimes required.
(1) $T D_{i+1, k}=T D_{i, k}+F r e q$
(2) $\left(T D_{i, k}+F r e q-\delta\right) \leq T D_{i+1, k} \leq\left(T D_{i, k}+F r e q+\delta\right)$

- Departure interval for the departure of the first trains going in both the up and down directions.

$$
\text { StartTime }_{i}<T D_{i, 1}<\text { EndTime }_{i}
$$

3. Railway infrastructure topology and type of trains to be scheduled give rise to other constraints to be taken into account. Some of them are:
- Number of tracks in stations (to perform technical and/or commercial operations) and the number of tracks between two locations (one-way or two-way).

- Time constraints, between each two contiguous stations $\left(T_{i,\left(k_{-} k+1\right)}\right)$.

$$
T A_{i, k+1}-T D_{i, k}=T_{i,\left(k \_k+1\right)}
$$

Figure 11 shows the set of variables of two trains going in opposite directions between stations $A$ and $E$. After studying the problem, we have detected an advantageous tree partition of the railway scheduling problem. In Figure 11, the edges between two variables represent time constraints $\left(T D_{i, k^{-}}-T A_{i, k+1}\right)$ and stop time constraints $\left(T A_{i, k^{-}} T D_{i, k}\right)$, respectively; these constraints make up train paths and they could be private information of railway operators. Figure 12 shows a clear tree partition of the railway scheduling problem where each sub-CSP has all variables of only one train and the respective intra-constraints are time constraints and time stop constraints that are usually fixed by railway operators. The inter-constraints are the traffic rules that are usually controlled by infrastructure managers.

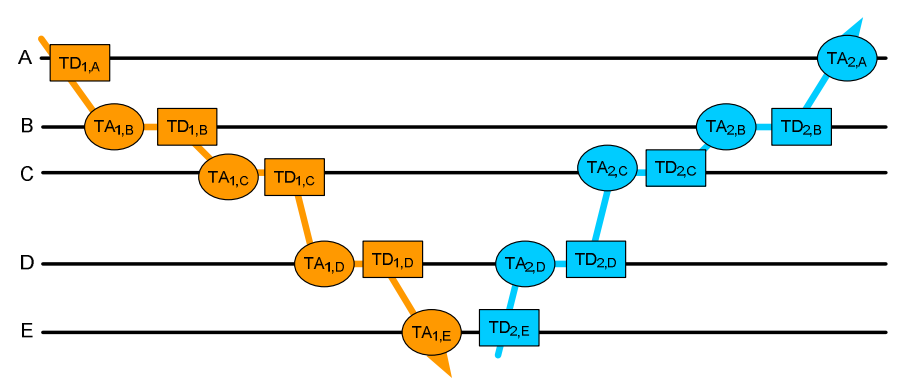

Figure 11: Variables of two opposite trains.

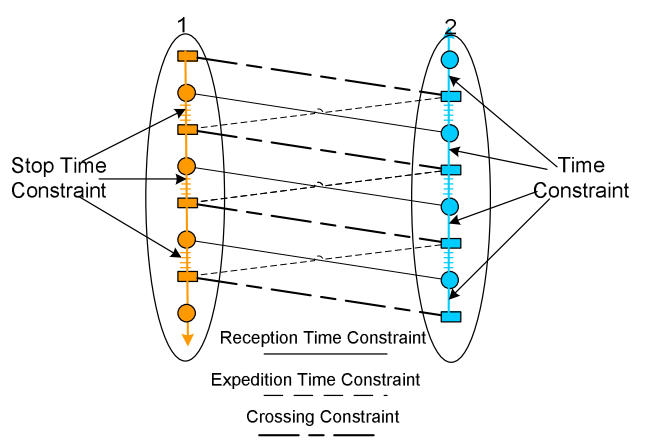

Figure 12: Train-based tree partition.

\section{Evaluation}

In this section, we carry out an evaluation between DTS and a centralized CSP solver. To this end, we have used a well-known centralized CSP solver called Forward Checking (FC). The classical binary version of FC ((Haralick \& G. 1980)) has a prohibitive computational cost for the type of problems evaluated in this section (a simple railway scheduling problem with 4 trains could not be solved after 1 day of 
execution); that is why we use the full path consistency Forward Checking algorithm (FCPath) ${ }^{1}$. This algorithm performs full path consistency on future variables whenever the current variable is instantiated.

This empirical evaluation of the railway scheduling problem was carried out over a real railway infrastructure that joins two Spanish cities (La Coruna and Vigo). The journey between these two cities is currently divided by 40 stations. In our empirical evaluation, each set of random instances was defined by the 3 -tuple $\langle n ; s ; f>$, where $n$ was the number of periodic trains in each direction, $s$ the number of stations, and $f$ the frequency (in minutes). The problems were randomly generated by modifying these parameters. Usually, increasing the number of trains involves a CSP with a greater number of variables; increasing the number of stations involves a CSP with a greater number of variables and a greater domain size; and decreasing the frequency involves increasing the problem tightness because the number of conflicts between trains is greater.

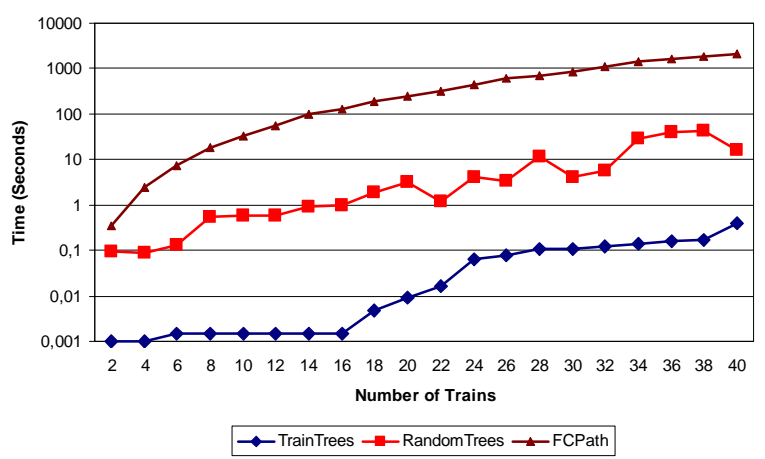

Figure 13: Running Times in problems $<n, 5,60\rangle$.

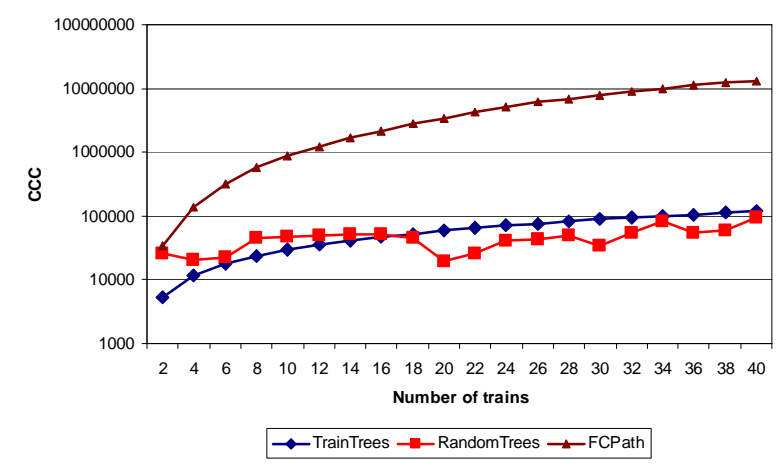

Figure 14: Concurrent constraint checks in problems $<n, 5,60>$.

In this evaluation, we compare the running time and concurrent constraint checks (CCC) ((Meisels et al. 2002)) of DTS with a well-known centralized algorithm: FCPath. DTS is executed over two different tree partitions: random

\footnotetext{
${ }^{1}$ FCPath was obtained from Van Beek page. It can be found in: http://ai.uwaterloo.ca/ vanbeek/software/software.html
}

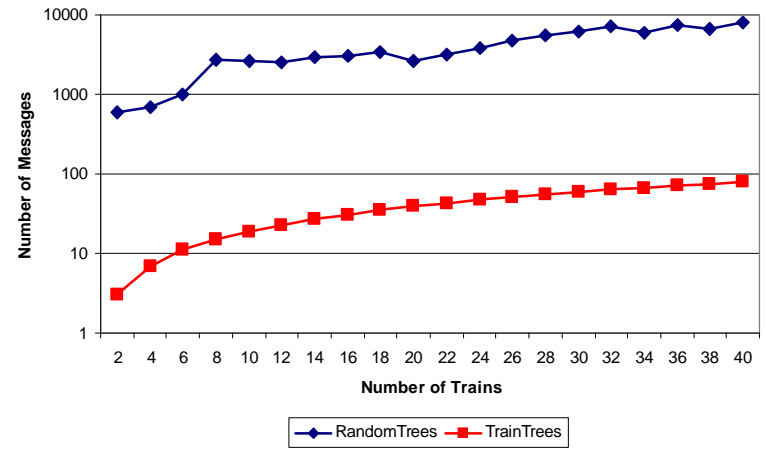

Figure 15: Message exchanges in problems $<n, 5,60>$.

partition is obtained using the TreePartition algorithm (Algorithm 1), which is a general tree partition method; train partition is a domain-dependent partition, which has been illustrated in Figure 12. It must be taken into account that both types of partitions roughly generate the same number of sub-problems and inter-constraints.

Figures 13 and 14 show the behaviors of DTS and FCPath in several instances of $n$ according to the tuple $\langle n, 5,60\rangle$. The number of trains $(n)$ was increased from 1 to 20 trains in each direction. It must be taken into account that both graphs maintain a $\log 10$ scale. Figures 13 and 14 show that DTS outperforms the FCPath algorithm, both random partition and train partition, in all instances. Figure 13 shows that DTS with train partition always has smaller running times than DTS with random partition. However, Figure 14 shows that DTS with random partition sometimes has fewer CCC than DTS with train partition. The last two assertions seem to be contradictory, but the explanation is in Figure 15: DTS with train partition exchanges fewer messages than DTS with random partition; thus, train partition saves a lot of running time. This is due to the fact that train partition involves better agent coordination.

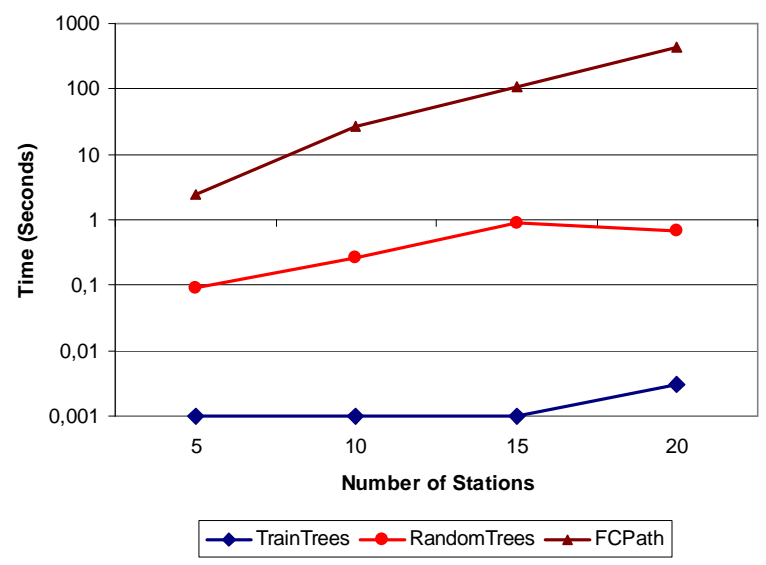

Figure 16: Running Times in problems $<4, s, 60\rangle$.

Figure 16 shows the behaviors of DTS and FCPath in several instances of $s$ according to the tuple $\langle 4, s, 60\rangle$, where the number of stations $(s)$ was increased from 5 to 20 . It can 


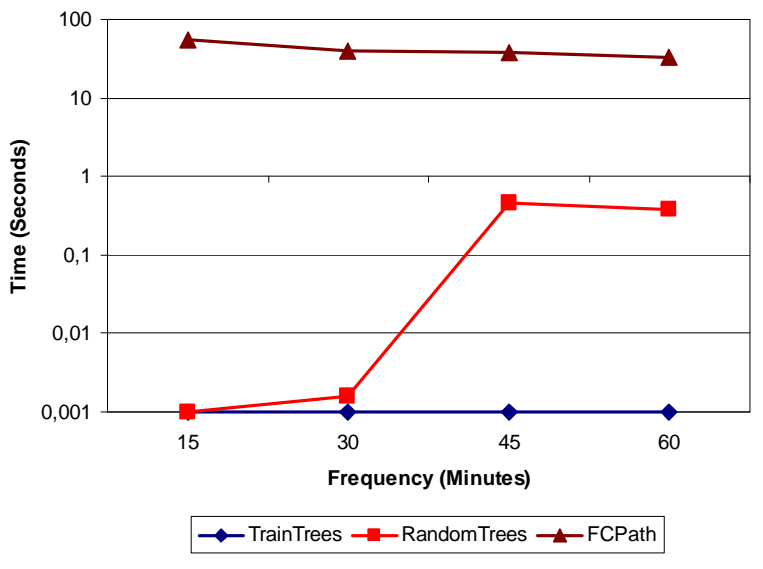

Figure 17: Running Times in problems $\langle 4,10, f\rangle$.

be observed that DTS with train partition has a better behavior than DTS with random partition and FCPath. In Figure 17 , we evaluate the behavior of the algorithms with different frequencies according to the tuple $\langle 4,10, f\rangle$, where frequency was increased from 15 to 60 minutes. It can be observed that sice the problem instances have the same number of variables and domain size, both DTS with train partition and FCPath have homogeneous behaviors. In general, both graphs corroborate the good behavior of the DTS algorithm, particularly with train partition.

\section{Conclusions}

We have presented three techniques for structuring and solving binary CSPs. The first one translates, in polynomial time, the original binary graph into a meta-tree CSP structure, where each node in the meta-tree CSP structure is a tree. The second technique is a distributed algorithm (DTS) for solving the resultant meta-tree CSP structure. DTS exploits the linear complexity to solve each tree and minimizes the storage of Nogoods. The third technique is an intraagent search algorithm. This algorithm takes into account the Nogood_message to prune the search space. These techniques have been applied to the railway scheduling problem. The evaluation shows that general distributed models have a better behavior than the centralized model and that domaindependent distributed models are more efficient than general ones. Thus, this technique may be appropriate for solving centralized problems that can be divided into smaller subproblems.

\section{Acknowledgements}

This work has been partially supported by the research projects TIN2007-29666-E and TIN2007-67943-C02-01 (Min. de Educacion y Ciencia, Spain-FEDER), FOM70022/T05 (Min. de Fomento, Spain), GV/2007/274 (Generalidad Valenciana) and by the Future and Emerging Technologies Unit of EC (IST priority - 6th FP), under contract no. FP6-021235-2 (project ARRIVAL).

\section{References}

Cordeau, J.; Toth, P.; and Vigo, D. 1998. A survey of optimization models for train routing and scheduling. Transportation Science 32:380-446.

Decher, R. 2003. Constraint Processing. Morgan Kaufmann.

Dechter, R., and Pearl, J. 1987. Network-based heuristics for constraint satisfaction problems. Artificial Intelligence 34:1-38.

Dechter, R. 1992. Constraint networks (survey). Encyclopedia Artificial Intelligence 276-285.

Freuder, E. 1982. A sufficient condition for backtrack-free search. Journal of the ACM 29:24-32.

Haralick, R., and G., E. 1980. Increasing tree efficiency for constraint satisfaction problems. Artificial Intelligence 14:263-314.

Meisels, A.; Kaplansky, E.; Razgon, I.; and Zivan, R. 2002. Comparing performance of distributed constraint processing algorithms. In Proc. 4th Workshop on Distributed Constraint Reasoning.

Miller, G. 1986. Finding small simple cycle separators for 2-connected planar graphs. Journal of Computer and System Sciences 32:265-279.

Salido, M., and Barber, F. 2006. Distributed CSPs by graph partitioning. Applied Mathematics and Computation 183:491-498.

Schloegel, K.; Karypis, G.; and Kumar, V. 2003. Graph partitioning for high-performance scientific simulations. Sourcebook of parallel computing 491-541.

Schrijver, A., and Steenbeek, A. 1994. Timetable construction for railned. Technical Report, CWI, Amsterdam, The Netherlands.

Serafini, P., and Ukovich, W. 1989. A mathematical model for periodic scheduling problems. SIAM Journal on Discrete Mathematics 550-581.

Silva de Oliveira, E. 2001. Solving single-track railway scheduling problem using constraint programming. Phd Thesis. Univ. of Leeds, School of Computing.

Walker, C., S. J., and Ryan, D. 2005. Simultaneous disruption recovery of a train timetable and crew roster in real time. Comput. Oper. Res 2077-2094.

Yokoo, M., and Hirayama, K. 2000. Algorithms for distributed constraint satisfaction: A review. Autonomous Agents and Multi-Agent Systems 3:185-207. 